\title{
Classification of Human Poses using a Vision based Technique
}

\author{
Dan Kelly, Paolo Olivo, Charles Markham, John McDonald, Dept. CS, NUI Maynooth \\ Brian Caulfield, Diarmuid Fitzgerald, Dept. Physiotherapy, UCD, Dublin \\ dankelly@cs.nuim.ie
}

\begin{abstract}
This paper presents work being carried out to estimate human pose using vision based methods. The data acquisition system uses an active marker technique synchronized with a three camera stereo vision system. The locations of the markers are then used to reconstruct a skeleton representation of the human pose. PCA and clustering techniques are used to classify the pose.
\end{abstract}

\section{Introduction}

Motion capture (MoCap) provides a technique to retrieve a set of parameters that can formally describe a subject's movements in a scene. The proposed system describes the pose of the human body by defining the position of a set of active markers placed on various parts of a subjects' body.

Using this tracking method we propose a system that can classify different human poses using PCA (principal component analysis) and clustering techniques. PCA is used to reduce the pose dimensionality and thus allows each pose to be described by a 3 dimensional vector. Low complexity clustering techniques can then be applied to these pose vectors to classify different types of pose.

\section{Data acquisition}

Body points are defined by white LEDs attached to the subject. The markers are turned on in sequence synchronized with the cameras. This approach simplified the correspondence problem.

\section{Data processing}

Data acquisition provided 2D coordinates of each LED through time for each camera. Using the $2 \mathrm{D}$ with the calibration matrix data provides the $3 \mathrm{D}$ coordinates of each point [1]. To obtain calibration matrices T. Svoboda's calibration toolbox [2] was used. These points were then used to reconstruct pose information (see figure 1).

\section{Pose Classification}

To classify poses the system uses the 45 dimensional feature vectors describing the poses. Using PCA the dimensionality of the pose description is reduced to 3. Experiments show that on average
$91 \%$ of pose information is retained in the dimension reduction.

Supervised learning is applied to the classifier using known poses. PCA is applied to known pose data then clustering each set of known poses and labeling each cluster accordingly (see figure 2). Pose recognition is then achieved by performing PCA on the subject's current pose and examining the location of the reduced vector in relation the center of gravity of the desired pose cluster. Eleven data sets constituting 5 poses were studied.

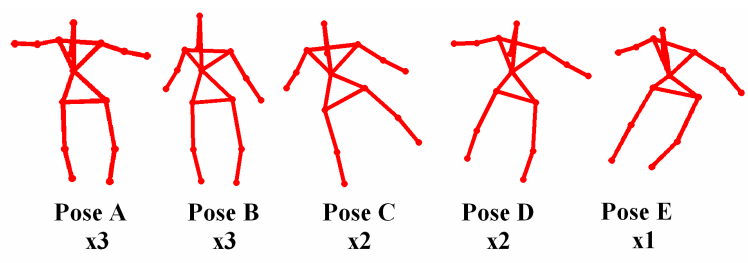

Figure 1: 3D reconstruction of the 5 different poses performed

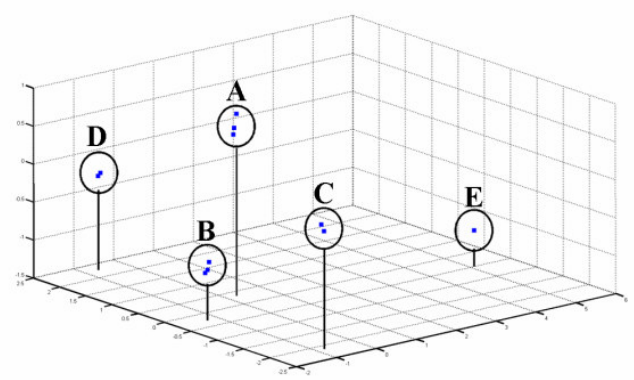

Figure 2: Pose Clusters in PCA space

\section{References}

[1] E. Trucco, A. Verri, Introductory techniques for 3D computer vision, Prentice-Hall, 1998.

[2] T. Svoboda, D. Martinec, and T. Pajdla. "A convenient multi-camera self-calibration for virtual environments". PRESENCE: Teleoperators and Virtual Environments, 14(4), August 2005. 